\title{
Humans socially attune to their "follower" robot
}

\author{
Francesca Ciardo \\ Social Cognition in Human-Robot \\ Interaction \\ Italian Institute of Techology \\ Genoa, Italy \\ francesca.ciardo@iit.it
}

\author{
Davide De Tommaso \\ Social Cognition in Human-Robot \\ Interaction \\ Italian Institute of Techology \\ Genoa, Italy \\ davide.detommaso@iit.it
}

\author{
Agnieszka Wykowska \\ Social Cognition in Human-Robot \\ Interaction \\ Italian Institute of Techology \\ Genoa, Italy \\ agnieszka.wykowska@iit.it
}

\begin{abstract}
In this study, we examined if humans adapt their performance to delays in robot's actions in a leaderfollower interaction scenario. Participants were asked to "teach" a sequence of musical tones to the iCub robot. The robot repeated the sequence with decreasing delay between its own taps and taps performed by the participants. We observed that mean period of participants' tapping behavior was affected by the iCub's performance. This suggests that humans are sensitive to subtle parameters in robot's behavior and they adapt to them in leader-follower contexts.
\end{abstract}

Keywords—social attunement; joint action; human-robot interaction

\section{INTRODUCTION}

Effective human-robot interactions (HRI) rely on the possibility of humans to socially attune to artificial agents [1]. When humans act in social contexts, the presence of another agent influences their performance at the sensorimotor level $[2,3]$. Several studies showed that during leader-follower joint task, as teaching a task to a pupil, humans adapt their action programs and execution to their follower [e.g. 4, 5]. Studies investigating how pedagogical contexts affect action programming showed systematic modulation of kinematics parameters. For instance, parents modulate the kinematics of their actions to highlight the significance of particular action elements and to communicate knowledge of the structure of actions to their infants [6]. The majority of the studies that investigated leader-follower tasks in HRI focused on how (and if) a robot learns from sensorimotor patterns of the human behaviour [e.g., 7, 8]. However, sensorimotor adaptation during joint task is a bidirectional phenomenon based on sensorimotor contingencies. For example, when we teach to a child how to bounce a ball, we start showing a simple and slow movement. As soon as we detect an improvement in pupil's performance, we increase firstly the speed and then the complexity of our demonstration. The present study aimed at investigating if humans adapt to robots' sensorimotor improvements during a leader-follower task. To this end, we designed a task in which participants (leaders) were required to teach iCub (follower) to play a simple melody consisting of 6 tones. During the task, we systematically decreased the delay between iCub taps and those of the human teacher.

\section{MATERIALS AND METHOD}

\section{A. Participants}

Seven healthy adults (mean age $=29.6 \pm 3.8 ; 2$ Males; 2 left-handed) took part in the study. The study was approved by the local ethical committee (Comitato Etico Regione Liguria).

\section{B. Experimental setup, stimuli and apparatus}

Our study was implemented in a multi-modal humanrobot interactive scenario, where the participant and the robot interacted with a vertical touch screen by producing a set of periodic audio sequences. The experimental setup consisted of a PC controlling the stimuli and responses, an iCub humanoid robot $[9,10]$, and a touchscreen. The multi-touch screen is 49 inches large (1099.4 x $634.0 \times 36.8 \mathrm{~mm})$, able to detect up to 32 touches, with a maximum resolution of 3840 x 2160 (4K UHD) and refresh rate up to $60 \mathrm{~Hz}$. The height of the touchscreen has been selected based on the iCub's arm position. The pointing gestures of the iCub robot were predefined movements achieved using joints positions as desirable targets in input to a direct position control algorithm. The default trajectory time of the robot's movements was $350 \mathrm{~ms}$. Accordingly, we empirically found the value of the sound duration of a single note $(450 \mathrm{~ms})$, which represents also the minimum period achievable of the audio sequence.

\section{Procedure}

Participants were presented with a music pad consisting of six different colored dots (diameter of each dot: $5 \mathrm{~cm}$ ) positioned on the vertices of a hexagon, to be all equidistant from the center (see Fig. 1). Each dot corresponded to a specific tone. iCub was positioned side by side of a participant, with its own pad consisting also of six colored dots. Participants were instructed to play a predefined melody of six different tones by touching on individual dots sequentially. They were all instructed to play the same melody, trying to keep a constant rhythm by tapping the dots in a circular way. In the training phase, participants played the melody until they performed ten repetitions of the sequence in a row. In the experimental phase, participants were instructed to teach the melody to iCub. Thus, while they were playing the sequence, iCub performed the same task as a follower. At the beginning of each trial the pad was presented as inactive (i.e. all the dots were inactive) with a central white dot. In order to start the trial, participants had to press the central dot and then, after releasing, all the dots of the pad were active and the participant could start playing the melody. Each trial ended after four repetitions of the melody,

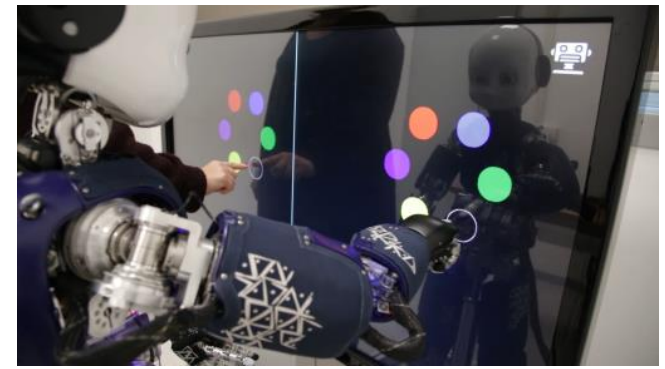

Fig.1 Example of an experimental trial (delay condition: $450 \mathrm{~ms}$ ). 
i.e. after twenty-four button presses taps (6 tones $\mathrm{x} 4$ repetitions) in the correct order. If a participant made a mistake, the trial was aborted. In total, participants performed 28 trials. Across trials we manipulated the average delay of iCub's performance (delay condition) in tapping the respective dots (corresponding to those tapped by the human) on its own pad. In the first 8 trials, iCub performed with a delay of $650 \mathrm{~ms}$. Then, in the 8 subsequent trials iCub's performance was delayed by $550 \mathrm{~ms}$. In the last 12 trials iCub tapped on each dot with a delay of $450 \mathrm{~ms}$. These delays represent the iCub's response time for tapping a single dot, that is, the time between the touches of the participant and the touches of iCub detected from the touchscreen for the respective dot on iCub's side. The delays have been selected considering the trajectory time of iCub movements which is $350 \mathrm{~ms}$ and a safety range of $100 \mathrm{~ms}$ due to the variability of the position controller. iCub reacted merely by repeating participant's actions. Once a dot selection is detected, the task controller sends a request to the robot for tapping the same dot. In this phase of the experiment, we decided not to add self-generated mistakes in the performance of the robot. Participants were not informed about how iCub's performance was programmed. For each trial we collected the following dependent measures: average period of the touch behaviour and its variance. We estimated the period as the mean of all the time differences between two consecutive taps of the participant. Moreover, in each trial we estimated the phase as the amount of time between the release of the central white dot and the touch on the first dot of the sequence.

\section{RESULTS}

Means of average period performance, period variance, and trial phase were submitted to a repeated-measures analysis of variance (ANOVA) with delay Condition (650 $\mathrm{ms}, 550 \mathrm{~ms}, 450 \mathrm{~ms}$ ) as within-subjects factor. When necessary, comparisons were performed using pairedsamples t-tests. The analysis revealed a significant effect of delay condition on period performance, $F(1,6)=3.99$, $p<$ $0.047, \eta_{p^{2}}{ }^{2}=0.40$. Paired sample t-test showed that participants' average period performance was lower when iCub performed the task with a shorter delay (i.e. $450 \mathrm{~ms}$ ) compared to when it acted with $650 \mathrm{~ms}$ delay, $\mathrm{t}(6)=2.48, \mathrm{p}$ $=0.048, d^{\prime}=0.92$. No other comparison was significant (all ps $>0.13$ ), see Fig. 2. Neither period variance nor trial phase (all Fs $<1$ ) were affected by the delay manipulation.

\section{CONCLUSIONS}

In this study we investigated if humans are able to detect and adapt to changes in iCub's sensorimotor contingencies. To this end, we designed a leader-follower task in which participants (leaders) were required to teach iCub (follower) to play a melody. During the task, we systematically manipulated the delay in sensorimotor contingency of $\mathrm{iCub}$ performance. Results showed that participants adapted their own actions to iCub's performance, thereby showing sensitivity to iCub's performance improvements. Future studies might include explicit measures to assess if the improvements in performance of the artificial agent affect how it is perceived socially. Our results highlight the bidirectional nature of human-robot adaptation during joint tasks, showing that when humans interacts with a follower humanoid, they socially attune with the artificial agent at the sensorimotor level. Such a results underlie the importance of

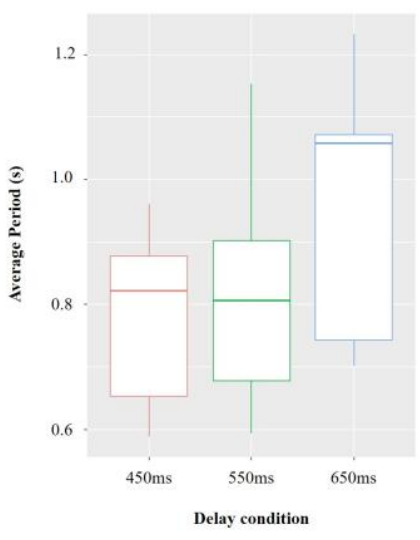

Fig.2 Average period performance plotted as a function of delay condition: $650 \mathrm{~ms}$ (blue). $550 \mathrm{~ms}$ (green). and $450 \mathrm{~ms}$ (red).

focusing on the "h" (human) side of HRI. If we imagine a robot interacting with a human in a work place, it appears crucial that robot's behavior should be designed so that it does not affect human's performance in a detrimental manner. For example, if the robot is to slow in performing its own task, the human co-agent might be also affected, resulting into a slowing down of the entire process.

\section{ACKNOWLEDGMENT}

This project has received funding from the European Research Council (ERC) under the European Union's Horizon 2020 research and innovation programme (grant awarded to A. Wykowska, titled "InStance: Intentional Stance for Social Attunement. Grant agreement No: 715058).

\section{REFERENCES}

[1] A. Wykowska, T. Chaminade, G. Cheng, "Embodied artificial agents for understanding human social cognition". Phil. Trans. Roy. Soc. B, vol. 371, pp. 1-9, May 2016. doi: 10.1098/rstb.2015.0375

[2] F. Ciardo, A. Wykowska, "Response Coordination Emerges in Cooperative but Not Competitive Joint Task". Front Psychol., vol 9, pp. 1-15, Oct 2018. doi: 10.3389/fpsyg.2018.01919.

[3] T. Lorenz, A. Mortl, and S. Hirche, "Movement synchronization fails during non-adaptive human-robot interaction". in 8th ACM/IEEE International Conference on Human-Robot Interaction (HRI), pp. 189-190, March. 2013. doi: 10.1109/HRI.2013.6483565

[4] M. J. Richardson, K. L. Marsh, R. W. Isenhower, J. R. L. Goodman, and R. C. Schmidt, "Rocking together: dynamics of intentional and unintentional interpersonal coordination". Hum. Mov. Sci., vol. 26, no. 6, pp. 867-91, Dec. 2007.

[5] G. Pezzulo, F. Donnarumma, and H. Dindo, "Human sensorimotor communication: a theory of signaling in online social interactions". PloS. One, vol. 8, no. 11, p. e79876, Jan. 2013.

[6] R. J. Brand, D. A. Baldwin, L. A. Ashburn, "Evidence for 'motionese': Modifications in mothers' infant-directed action". Developmental Science, vol. 5, pp. 72-83, Aug. 2009. doi: 10.1111/1467-7687.00211

[7] E. Gribovskaya, A. Kheddar, A. Billard,"Motion learning and adaptive impedance for robot control during physical interaction withhumans". In IEEE International Conference on Robotics and Automation (ICRA), pp. 4326-4332, May 2011.

[8] A.Vignolo, N. Noceti, F. Rea, A. Sciutti A., F. Odone F, G. Sandini, "Detecting biological motion for human-robot interaction: a link between perception and action”. Front. Robot. AI., vol. 4, Jun. 2017

[9] G. Metta, G. Sandini, D. Vernon, L. Natale, F. Nori, "The iCub humanoid robot: an open platform for research in embodied cognition". In Proceedings of the 8th workshop on performance metrics for intelligent systems, pp. 50-56, Aug. 2009

[10] L. Natale, C. Bartolozzi, D. Pucci, A. Wykowska, G. Metta, "The notyet-finished story of building a robot child”.Science Robotics, vol. 2, pp. eaaq1026, Dec. 2017. doi: 10.1126/scirobotics.aaq1026 